\title{
Computational Analysis of Interaction between Mycobacterial Antigens Rv0679c and Rv0180c with Toll like Receptors of Human and Mouse
}

\author{
Rupa Lavarti \\ Department of Biotechnology, \\ Sreenidhi Institute of \\ Science \& Technology, \\ Yamnampet, Ghatkesar, \\ Hyderabad- 501301, India \\ Shirisha Ratcha \\ Department of Biotechnology, \\ Sreenidhi Institute of \\ Science \& Technology, \\ Yamnampet, Ghatkesar, \\ Hyderabad- 501301, India
}

\author{
Jayasree Ganugapati \\ Department of Biotechnology, \\ Sreenidhi Institute of \\ Science \& Technology, \\ Yamnampet, Ghatkesar, \\ Hyderabad- 501301, India \\ Krovvidi S. R. SivaSai \\ Department of Biotechnology, \\ Sreenidhi Institute of \\ Science \& Technology, \\ Yamnampet, Ghatkesar, \\ Hyderabad- 501301, India
}

\begin{abstract}
Toll like receptors (TLRs) play a key role in the innate immune response to infectious agents. The Mycobacterium tuberculosis bacilli and its antigens interaction with TLR-2-1 and TLR-4-MD2 were shown to activate intracellular signalling which determines the outcome of the disease. Present study focused on two new antigens Rv0679c and Rv0180c of M.tb H37Rv and their interactions with human and mouse TLRs using computational approach. Structures of $\mathrm{Rv} 0679 \mathrm{c}$ and Rv0180c antigens of M.tb were generated using I-TASSER and docked with TLR-2-1 and TLR-4-MD2 complexes of human and mouse using Cluspro2.0. Rv0180c antigen has better binding energy to both human and mouse TLR-2-1 and TLR-4-MD2 compared to Rv0679c and other mycobacterial antigens. The Rv0679c and Rv0180c antigens have better binding energy to TLR 4-MD2 complex compared to TLR-2-1 in both human and mouse. Both antigens has better binding energy to mouse TLRs compared to human except where Rv0180c has better binding to human TLR-2-1. Our findings suggest that Rv0180c might be the preferred for binding to TLRs than Rv0679c and within TLRs, TLR-4MD2 for interaction.
\end{abstract}

\section{General Terms}

Invasive mechanism of mycobacterial antigens using TLRs

\section{Keywords}

Mycobacterium tuberculosis, toll like receptors, invasion, ITASSER, docking, ClusPro

\section{INTRODUCTION}

Intracellular pathogen Mycobacterium tuberculosis (M.tb) even though the earliest to be indentified which causes disease in humans still remain a major global health threat [1] One of the reasons could be development of effective evasion strategies adopted by bacterium to overcome host defence mechanisms [2]. Sequencing of whole genome of bacterium [3] lead to better understanding of membrane bound antigens associated with host receptor interaction leading to probable invasion / entry into the host.
Two novel proteins Rv0679c and Rv0180c were identified from M.tb H37Rv, which are membrane bound and play a role in entry / invasion of pathogen into host cells [4, 5]. Rv0679c protein was classified as a membrane protein of the envelope of M.tb with a molecular mass of $16.5 \mathrm{KDa}$, consisting of 165 amino acids and contains a putative $\mathrm{N}$-terminal signal sequence and a consensus lipoprotein-processing motif. Rv0679c protein exists as a tight complex with LAM, one of the major components of cell envelope involved in proinflammatory and anti-inflammatory responses [6]. Cifuentes et al [4] have shown that peptides of Rv0679c inhibited invasion / enrty of live M.tb bacilli into A549 and U937 macrophage cell lines in vitro. Rv0180c is a conserved transmembrane protein with a molecular mass of $47.5 \mathrm{KDa}$, consisting of 452 amino acids with unknown function however shares a high sequence similarity with putative conserved membrane and transmembrane proteins from different mycobacterial species [5]. Caceres [5] have shown that the peptides of this protein inhibited the invasion of M.tb H37Rv into U937 and A549 cell lines in vitro indicating its candidature as a subunit based vaccine against tuberculosis. Either Rv0679c or Rv0180c protein were not evaluated for the possible mechanism of blocking invasion into the cells, which could be probably through specific receptors.

Pattern recognition receptors (PRRs) like toll-like receptors (TLRs) play a pivotal role in the induction of an innate immune response to various infectious agents, including mycobacteria spp [7]. The interface between M.tb and its proteins to various TLRs is not fully understood, but it appears that whole mycobacteria or distinct mycobacterial components may interact with different members of the TLR family [8]. Studies indicated that TLR2 and TLR4 play a major role in regulating innate recognition to mycobacterial infection [9]. Encounter with triacylated or diacylated lipoproteins induces heterodimerization of human TLR-2 either with TLR-1 or TLR-6 [10]. Similarly TLR-4, associates with Myeloid differentiating factor (MD-2) and it is believed to be the component of the TLR4-MD-2 complex that interacts with lipopolysaccharides of different bacteria [11]. 
TLRs are highly conserved from Drosophila to humans and share structural and functional similarities. Mouse is being used as a model to study the pathogenesis of tuberculosis for better understanding of mechanisms related to bacterial pathogenicity vs. host resistance and susceptibility. Hence in the present study simultaneous assessment of the interaction of mycobacterial proteins with human and mouse TLRs was done for comparative analysis. Three different mycobacterial proteins such as LprG, Ag85 and MPT51 were used for comparison.

Antigen 85 complex (Ag85) of M.tb consists of three abundantly secretary proteins ( $\mathrm{FbpA}, \mathrm{FbpB}$ and $\mathrm{FbpC} 2$ ) which play a role in the pathogenesis of tuberculosis apart from exhibiting cell wall mycolyl transferase activity [12]. MPT51, a secretary protein of M.tb shares $40 \%$ identity with the Ag85 components and is involved in cell wall mycoloylation [13]. LprG is a conserved membrane Lipoarabinomannan carrier protein essential for virulence of M.tb [14]. LprG binding to LAM facilitates its transfer from the plasma membrane into the cell envelope, increasing surface-exposed LAM, enhancing cell envelope integrity, allowing inhibition of phagosome-lysosome fusion and enhancing M.tb survival in macrophages [15].

The combined approach of proteomics, protein-protein interaction and receptor-ligand interaction studies using bioinformatics approach has potential to generate information and knowledge that will enable in understanding hostpathogen interaction leading to entry / invasion into host cell. This enables the development of new therapies and interventions strategies needed to treat or control disease. In the light of above the present study assessed whether the Rv0679c and Rv0180c hypothetical invasive proteins of M.tb could bind to TLRs and if so might be associated as molecules for entry in humans and mouse system.

\section{METHODOLOGY}

\subsection{Retrieval of Rv0679c and Rv0180c sequences and Homology modeling using I-TASSER}

The sequence of Rv0679c (165 aminoacids) and Rv0180c (498 aminoacids) antigens were retrieved from TubercuList database [http://tuberculist.epfl.ch].Structures of Rv0679c and Rv0180c antigenss were generated using I-TASSER. $[16,17,18]$. Iterative Threading ASSEmbly Refinement (I-TASSER) is a hierarchical method for protein structure and function prediction. Briefly, the structures are generated by LOMETS (Local Meta-Threading-Server) first, which further enables to generate 3D models by collecting high-scoring target-to-template alignments from 9 locally-installed threading programs. Atomic models are then constructed by iterative template fragment assembly simulations; functional insights of the target are finally derived by threading the 3D models through protein function database BioLiP.

\subsection{Retrieval of M.tb antigen structures}

Structures of other mycobacterial antigens like LprG (PDB ID: 3MH8), Ag85 (PDB ID: 1SFR), MPT51 (PDB ID: 1R88) were obtained for comparative analysis from protein data bank (PDB) [http://www.rcsb.org/pdb/home/home.do]. The X-ray crystallographic structures of human TLR-2-1 heterodimer PDB (PDB id- 2z7x) and TLR-4-MD-2 dimers (PDB ID: 3FXI) and mouse TLR-2 (PDB Id: 2Z82) and TLR4-MD2 (PDB Id: 2Z64) dimer were also retrieved from PDB.

\subsection{Structure validation using PROCHECK}

Structures of Rv0679c and Rv0180c antigens generated by I-TASSER was validated using PROCHECK, which is available online at http://www.ebi.ac.uk/thornton-srv/software /PROCHECK/ to ensure zero residues in the disallowed regions. It provides a detailed check on the stereochemistry of the protein structure. PROCHECK produces a number of PostScript plots, analyzing its overall and residue-by-residue geometry. The final models obtained were used for docking analysis.

\subsection{Docking Studies}

Docking of antigens with human and mouse TLR's was performed using Cluspro2.0 where in antigen is considered as ligand and TLR as a receptor [http://cluspro.bu.edu /home.php]. Cluspro2.0 is a web-based automated program for the computational docking of protein structures. Once the coordinate files of the protein structures are uploaded the docking algorithms evaluate billions of putative complexes, retaining a preset number with favourable surface complementarities. The obtained structures are then filtered, selecting those with good electrostatic and desolvation free energies for further clustering.

\section{RESULTS \& DISCUSSION}

\subsection{Prediction of 3D structures of Rv0679c and Rv0180c antigens of M.tb}

Structures of Rv0679c and Rv0180c antigens generated by ITASSER were submitted to PBDsum database (http://www.ebi.ac.uk/thornton-srv/databases/cgi-bin/pdbsum/ GetPage.pl?pdbcode=index.html) which provides an overview of the contents of the protein structures. Secondary structure analysis of Rv0679c using PDBSum revealed 2 helices, 16 beta turns and 7 gamma turns (Figure 1A) while Rv0180c secondary structure has 25 helices, 32 beta turns and 12 gamma turns (Figure 1B).
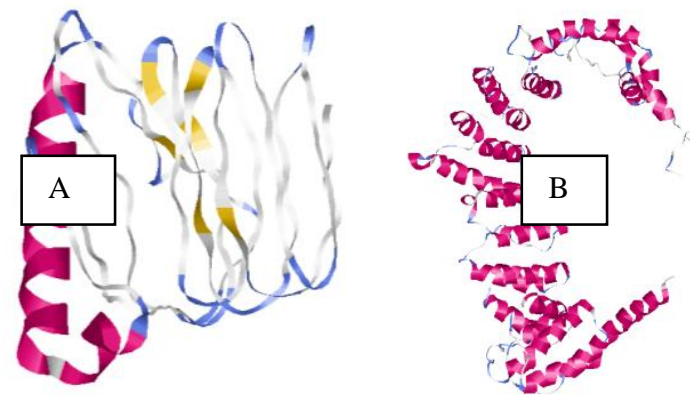

Figure 1: Structure of Rv0679c and Rv0180c antigens of M.tb generated using I-TASSER.

A: In Rv0679c antigen structure pink indicates alpha helix; yellow indicates beta sheets, blue indicates turns and white indicates coils. B: In Rv0180c antigen structure Pink indicates alpha helix and blue indicates turns.

\subsection{Validation of antigen structures}

Structures of Rv0679c and Rv0180c antigens generated by

I-TASSER were validated using PROCHECK. Figure 2 depicts Ramachandran plot for Rv0679c antigen with zero residues in the disallowed region. The Ramachandran plot for Rv0180c antigen is depicted in Figure 3, showing zero residues in the disallowed region. PROCHECK statistical data for both the antigens was shown in Table $1(\mathrm{Rv} 0679 \mathrm{c})$ and 
Table 2 (Rv0180c). This study is the first report on the structures of Rv0679c and Rv0180c antigens obtained using computational approach as the crystallography studies are not reported so far.

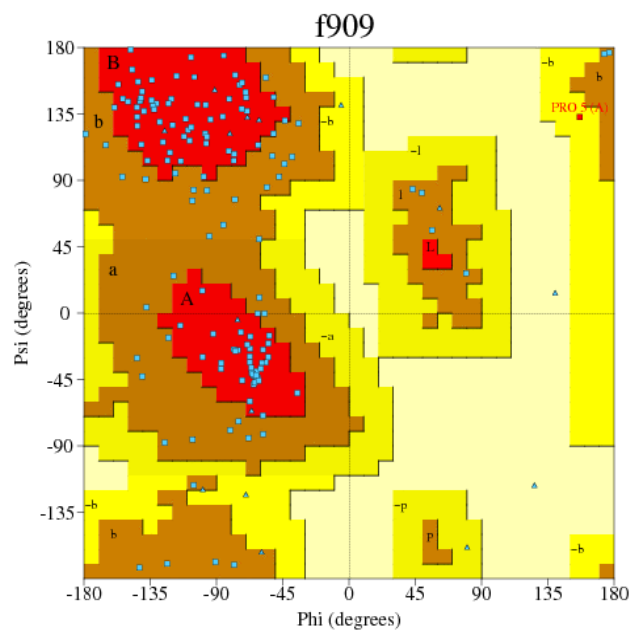

Figure 2: Ramachandran plot of Rv0679cantigen

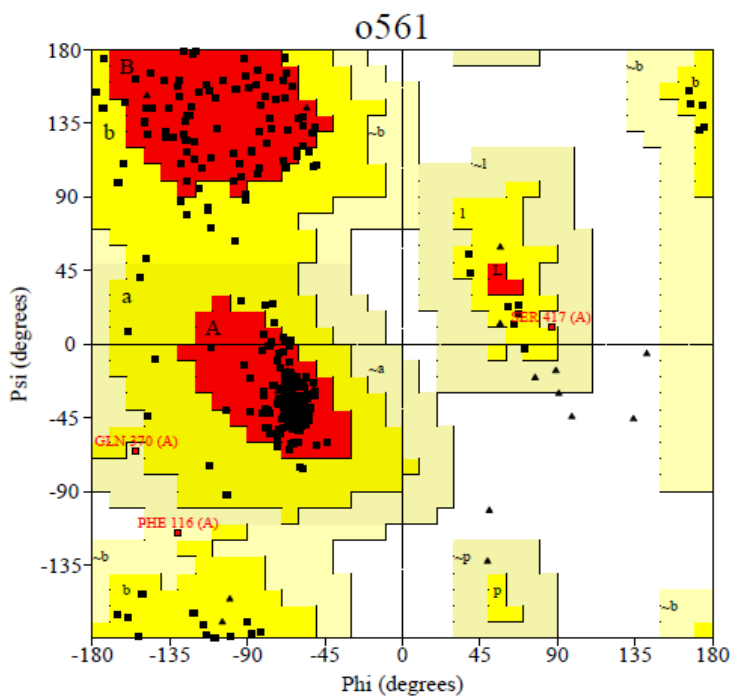

Figure 3: Ramachandran plot of Rv0180c antigen

Table 1 : PROCHECK statistics for Rv0679c antigen

\begin{tabular}{|c|c|c|}
\hline Ramchandran plot statistics & $\begin{array}{l}\text { No. of } \\
\text { residues }\end{array}$ & \%tage \\
\hline Most favoured regions [A,B,L] & 98 & 70 \\
\hline Additional allowed regions [a,b,l,p] & 42 & 30 \\
\hline Generouslyallowed regions & 0 & 0 \\
\hline Disallowed regions [xx] & 0 & 0 \\
\hline $\begin{array}{c}\text { Non-glycine and non-proline } \\
\text { residues }\end{array}$ & 140 & 100 \\
\cline { 1 - 2 } End-residues (excl, Gly and pro) & 2 & \multirow{2}{*}{100} \\
\hline Glycine residues & 15 & \\
\hline Proline residues & 8 & \\
\cline { 1 - 2 } Total number of residues & 165 & \\
\cline { 1 - 2 } &
\end{tabular}

\subsection{Docking analysis for assessment of binding energies}

TLR-2-1 and TLR-4-MD2 structures retrieved from PDB were docked with mycobacterial antigens. The binding energies and docked complexes obtained between human TLRs and antigens are depicted in Table- 3 and with mouse TLRs in Table- 4 .

Rv0180c and Rv0679c antigens has better binding energy of $1219 \mathrm{Kcal} / \mathrm{mol}$ and -988.6 to TLR-4-MD2 complex compared to TLR-2-1 with binding energy of $-1044 \mathrm{Kcal} /$ mol and -849.1 respectively in humans (Table 3). Rv0180c antigen has better binding energy compared to Rv0679c and other mycobacterial antigens like LprG, Ag85 and MPT51 used for comparative analysis (Table 3 ) for both TLR-2-1 and TLR-4-MD2. In general the antigens of mycobacteria preferentially had better binding affinity to human TLR-4MD2 compared to TLR-2-1, except in the case of LprG antigen.

In mouse, antigens Rv0180c and Rv0679c had better binding energy of $-1393.8 \mathrm{Kcal} / \mathrm{mol}$ and $-1138 \mathrm{Kcal} / \mathrm{mol}$ to TLR-4MD2 complex compared to TLR-2-1 with binding energy of $987.2 \mathrm{Kcal} / \mathrm{mol}$ and $-979.4 \mathrm{Kcal} / \mathrm{mol}$ respectively (Table-4). Rv0180c antigen has better binding energy compared to Rv0679c and other mycobacterial antigens like LprG, Ag85, MPT51 used for comparative analysis (Table-4)

for both TLR-2-1 \& TLR-4-MD2. This indicates that antigens

Table 2: PROCHECK statistics for Rv0180c antigen

\begin{tabular}{|c|c|c|}
\hline Ramchandran plot statistics & $\begin{array}{c}\text { No. of } \\
\text { residues }\end{array}$ & \%tage \\
\hline Most favoured regions $[\mathrm{A}, \mathrm{B}, \mathrm{L}]$ & 324 & 84.6 \\
\hline Additional allowed regions $[\mathrm{a}, \mathrm{b}, \mathrm{l}, \mathrm{p}]$ & 56 & 14.6 \\
\hline Generouslyallowed regions & 3 & 0.8 \\
\hline Disallowed regions $[\mathrm{xx}]$ & 0 & 0.0 \\
\hline $\begin{array}{l}\text { Non-glycine and non-proline } \\
\text { residues }\end{array}$ & 383 & 100 \\
\hline End-residues (excl, Gly and pro) & 1 & \\
\hline Glycine residues & 41 & \\
\hline Proline residues & 27 & \\
\hline Total number of residues & 452 & \\
\hline
\end{tabular}

of mycobacteria preferentially had better binding affinity even to mouse TLR-4-MD2 compared to TLR-2-1 except in the case of LprG antigen.

TLRs are the type 1 transmembrane glycoproteins characterized by the presence of an extracellular domain (ectodomain ECD) containing leucine rich repeats (LRR) which is primarily responsible for mediating ligand interaction [19]. Protein structure and nature of binding of mycobacterial proteins in the right pockets of TLRs determine / influence the outcome of interaction which could be beneficial or detrimental. Rv0180c protein was binding to TLR-2-1 and TLR-4-MD2 of humans in the conventional way interacting predominantly in the ectodomain and central domain of the receptor compared to other mycobacterial antigens which were binding to C-terminal end (Table-3) 
except in the case MPT51 and Rv0679c protein to TLR-4MD2. This suggests that membrane proteins such as Rv0180c, Rv0679c and MPT51 are binding in one pattern compared to the other two - Ag85 and LprG which are secretary proteins of M.tb. Antigen 85 was shown to interact with fibronectin domain regardless of specificity of the domain [20] and leads to enhancement of phagocytosis of M. leprae in monocytederived-macrophages [21]. Taken the findings of the present study, Ag85 binding to TLRs was non-conventional and Ag85 preferring fibronectin domain in case of mycobacteria as shown by Naito M [20], one would assume similar fibronectin domain interaction could be possible or preferred by M.tb.

Table 3: Docking of Mycobacterium tuberculosis antigens Rv0180c, Rv0679c, LprG, Ag85, MPT51 with human

TLR-2-1 Heterodimer and TLR-4-MD2 dimer

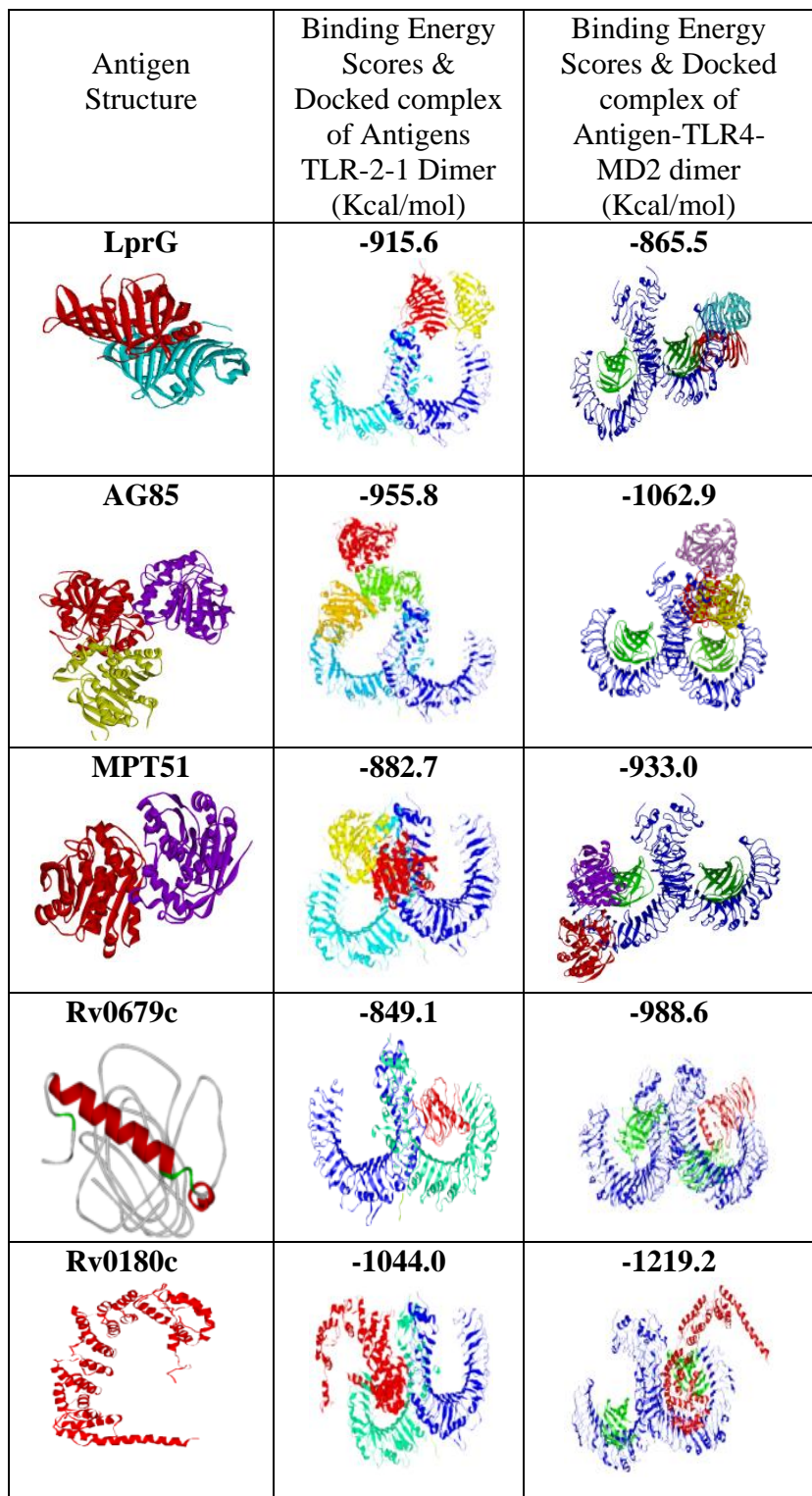

In TLR 2-1 docked complex dark blue indicates TLR-1; Sky blue and cyan indicates TLR-2. In TLR4-MD2 peptide docked complex dark blue indicates TLR4 dimers, while green indicates MD2.
Table 4: Docking of Mycobacterium tuberculosis antigens Rv0180c, Rv0679c, LprG, Ag85, and MPT51 with mouse TLR-2 and TLR-4.

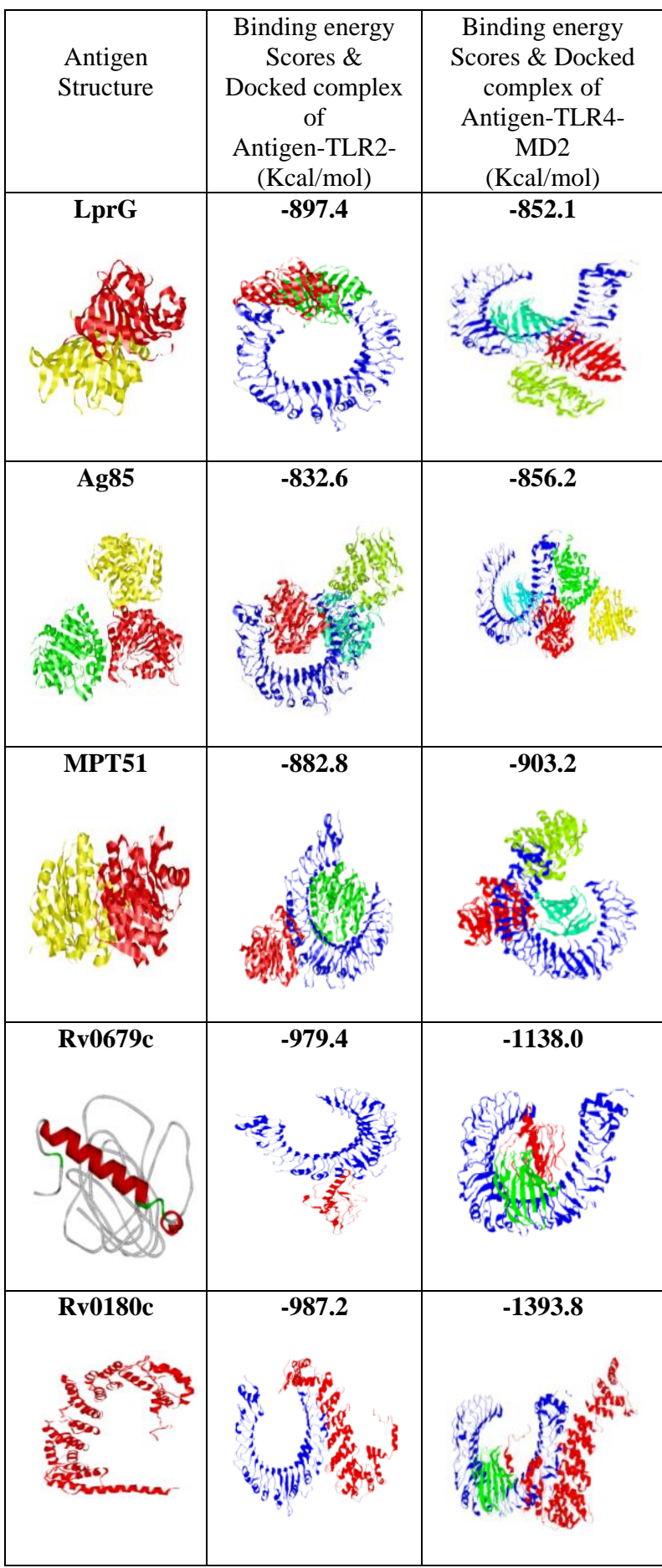

In TLR2 docked complex dark blue indicates TLR-2; In TLR4-MD2 peptide docked complex dark blue indicates TLR4 dimer, while green indicates MD2.

Stimulation studies using different lipoproteins of Mycobacteria such as LpqH (Rv3763) [22] , LprG (Rv1411c) [23], LprA (Rv1270c) [24] etc on murine bone marrow derived macrophages, human monocytic cell line and primary human macrophages suggested the ability of these proteins to inhibit the antigen processing, MHC expression and production of interferon-gamma in respective experiments. 
They observed that it was predominantly due to the interaction of these proteins with either TLR-2 or TLR-4 leading to redundant / diminished mechanisms affecting the recognition of infected macrophages by $\mathrm{T}$ cells and thus evading the immune system.

This study is the first report to indicate Rv0679c and Rv0180c proteins of M.tb interaction with TLRs, using computational approach. However, one could not comment on the functional outcome of Rv0679c and Rv0180c proteins interaction with TLRs, but speculate based on other lipoproteins study of M.tb. TLRs interaction with Rv0679c and Rv0180c proteins could lead to invasion / entry of pathogen inside the host subsequently modulating immune system for evading purpose.

\section{CONCLUSION}

The Rv0679c and Rv0180c proteins could bind to TLRs where the binding affinity / energies are high for human and mouse TLR-4 compared to TLR-2. Rv0180c protein has better binding energy than Rv0679c indicating Rv0180c might be more effective in invasion / entry compared to Rv0679c protein. The findings of both antigens interaction with TLRs in mouse and human are related; suggesting it could be a preferred model for addressing fundamental questions of TB pathogenesis in vivo.

\section{ACKNOWLEDGMENTS}

We express our in-depth thanks to Indian Council of Medical Research (ICMR) New Delhi, India for Senior Research Fellowship (SRF) assistance to Lavarti Rupa (File No: 80/747/2012-ECD-I). The group appreciates the support of management and administration of Sreenidhi Institute of Science and Technology and TEQIP-II program, World Bank Scheme, for the study.

\section{REFERENCES}

[1] Kaufmann, S.H, Hussey, G., Lambert, P.H. 2010. New vaccines for tuberculosis. Lancet 375: 2110-2119.

[2] Ottenhoff, T.H., 2009. Overcoming the global crisis: "yes, we can", but also for TB ... ? Eur J Immunol 39: 2014-2020.

[3] Cole, S.T, Brosch, R., Parkhill J. et al. 1998. Deciphering the biology of Mycobacterium tuberculosis from the complete genome sequence. Nature 393: 537-544.

[4] Cifuentes, D.P., Ocampo. M., Curtidor, H. 2010. Mycobacterium tuberculosis Rv0679c protein sequences involved in host-cell infection: Potential TB vaccine candidate antigen, BMC Microbiology, (4)10:109

[5] Cáceres, S.M., Ocampo, M., Arévalo-Pinzón G. et al. 2011. The Mycobacterium tuberculosis membrane protein Rv0180c: Evaluation of peptide sequences implicated in mycobacterial invasion of two human cell lines. Peptides 32:1-10.

[6] Matsuba, T., Suzuki, Y., Tanaka Y. 2007. Association of the Rv0679c protein with lipids and carbohydrates in Mycobacterium tuberculosis/ Mycobacterium bovis BCG. Archives of microbiology, 187(4):297-311.

[7] Takeda, K., and Akira S. 2001. Regulation of innate immune responses by Toll-like receptors. Jpn J Infect Dis. Dec; 54 (6):209-19.
[8] Eun-Kyeong Jo., Chul-Su Yang., Chul Hee Cho., and Clifford V. 2007. Harding Intracellular signalling cascades regulating innate immune responses to Mycobacteria: branching out from Toll-like receptors Cellular Microbiology, 9 (5):1087-1098.

[9] Means, T. K., Jones, B. W., Schromm, A. B. et al. 2001 Differential effects of a Toll-like receptor antagonist on Mycobacterium tuberculosis-induced macrophage responses. J. Immunol. 166:4074.

[10] Takeuchi, O., Sato S., Horiuchi T. et al. 2002. Cutting edge: role of Toll-like receptor 1 in mediating immune response to microbial lipoproteins. J. Immunol. 169: 10 14

[11] Shimazu, R., et al. 1999. MD-2, a molecule that confers lipopolysaccharideResponsiveness on Toll-like receptor 4. J. Exp. Med. 189, 1777-1782.

[12] Kremer, L., Maughan, W.N., Wilson, R.A. et al. 2002. The M. tuberculosis antigen 85 complex and mycolyltransferase activity. Lett Appl Microbiol. 34(4):233-7.

[13] Wilson, R.A., Maughan, W.N., Kremer, L. et al. 2004. The structure of Mycobacterium tuberculosis MPT51 (FbpC1) defines a new family of non-catalytic alpha/beta hydrolases. J Mol Biol. Jan 9;335 (2):519-30.

[14] Farrow, M.F., Rubin, E.J. 2008. Function of a mycobacterial major facilitator superfamily pump requires a membrane-associated lipoprotein. Journal of Bacteriology. 190(5):1783-1791.

[15] Supriya Shukla., Edward Richardson., Jaffre Athman. et al. 2014. Mycobacterium tuberculosis lipoprotein LprG binds lipoarabinomannan and determines its localization in the cell wall envelope and affects phagolysosomal fusion.The Journal of Immunology. vol.192 (1 Supplement) 132.18

[16] Yang, J., Yan R, Roy, A., Xu, D. et al. 2015.The ITASSER Suite: Protein structure and function prediction. Nature Methods, 12: 7-8.

[17] Roy, A., Kucukural, A., Zhang Y. 2010. I-TASSER: a unified platform for automated protein structure and function prediction. Nature Protocols, 5: 725-738.

[18] Zhang, Y. I-TASSER server for protein 3D structure prediction. BMC Bioinformatics, vol 9, 40 (2008).

[19] Kim, H. M., Park, B. S., Kim, J. I. et al. 2007. Crystal structure of the TLR4-MD-2 complex with bound endotoxin antagonist Eritoran. Cell 130, 90691710.1016/j.cell.2007.08.002

[20] Naito, M., Fukuda T., Sekiguchi, K., and Yamada T. 2000. The domains of human fibronectin mediating the binding of alpha antigen, the most immunopotent antigen of mycobacteria that induces protective immunity against mycobacterial infection. Biochem. J. 347, 725-731

[21] Schlesinger, L., S, and Horwitz, M. A. 1991. Phagocytosis of Mycobacterium leprae by human monocyte-derived macrophages is mediated by complement receptors CR1 (CD35), CR3 (CD11b/CD18), and CR4 (CD11c/CD18) and IFNgamma activation inhibits complement receptor function 
and phagocytosis of this bacterium. J. Immunol. 147, 1983-1994

[22] Noss, E. H., Pai, R.K., Sellati, T. J., et al. 2001. Tolllike receptor 2-dependent inhibition of macrophage class II MHC expression and antigen processing by $19 \mathrm{kD}$ lipoprotein of Mycobacterium tuberculosis. J. Immunol. 167: 910-918

[23] Gehring, A. J., Dobos, K. M., Belisle J. T. et al. 2004. Mycobacterium tuberculosis LprG (Rv1411c): a novel
TLR-2 ligand that inhibits human macrophage class II MHC antigen processing. J. Immunol.173:2660-2668.

[24] Pecora, N.D., Gehring, A.J., Canaday, D.H. et al. 2006. Mycobacterium tuberculosis LprA is a lipoprotein agonist of TLR2 that regulates innate immunity and APC function. J Immunol. Jul 1; 177(1):422-9. 